\title{
The WRKY70 transcription factor of Arabidopsis influences both the plant senescence and defense signaling pathways
}

\author{
Bekir Ülker • M. Shahid Mukhtar • Imre E. Somssich
}

Received: 25 September 2006 / Accepted: 21 December 2006 / Published online: 20 February 2007

(C) Springer-Verlag 2007

\begin{abstract}
Regulatory proteins play critical roles in controlling the kinetics of various cellular processes during the entire life span of an organism. Leaf senescence, an integral part of the plant developmental program, is fine-tuned by a complex transcriptional regulatory network ensuring a successful switch to the terminal life phase. To expand our understanding on how transcriptional control coordinates leaf senescence, we characterized AtWRKY70, a gene encoding a WRKY transcription factor that functions as a negative regulator of developmental senescence. To gain insight into the interplay of senescence and plant defense signaling pathways, we employed a collection of mutants, allowing us to specifically define the role of AtWRKY70 in the salicylic acid-mediated signaling cascades and to further dissect the cross-talk of signal transduction pathways during the onset of senescence in Arabidopsis thaliana. Our results provide strong evidence that AtWRKY70 influences plant senescence
\end{abstract}

B. Ülker · M. Shahid Mukhtar · I. E. Somssich ( $\square)$ Max Planck Institute for Plant Breeding Research, Abteilung Molekulare Phytopathologie, Carl-von-Linné-Weg 10, 50829 Cologne, Germany

e-mail: somssich@mpiz-koeln.mpg.de

Present Address:

B. Ülker

School of Biological and Biomedical Sciences,

Durham University, Science Site, South Road,

Durham DH1 3LE, UK

Present Address:

M. Shahid Mukhtar

Department of Biology,

University of North Carolina at Chapel Hill,

CB\# 3280, 108 Coker Hall, Chapel Hill, NC 27599, USA and defense signaling pathways. These studies could form the basis for further unraveling of these two complex interlinked regulatory networks.

Keywords Atwrky70 mutants · Dark-induced senescence $\cdot$ Salicylic acid $\cdot$ Signaling crosstalk
Abbreviations
ET Ethylene
JA Jasmonic acid
SA Salicylic acid

\section{Introduction}

Leaf senescence, constituting the terminal stage of leaf development, is a type of programmed cell death (PCD) and is manifested by a loss of chlorophyll that occurs during chloroplast disassembly together with other catabolic events such as protein, lipid and nucleic acids degradation (Quirino et al. 2000; Gepstein 2004). Leaf senescence is regulated by both intrinsic and environmental signals, with a number of phytohormones and growth regulators such as cytokinins, abscisic acid, salicylic acid, jasmonates, ethylene, nitric oxide and brassinosteroids acting in a precisely coordinated manner (Gepstein 2004). Although considered to be a highly entropic process, the onset, progression and completion of leaf senescence requires extensive and orchestrated transcriptional reprogramming, de novo protein synthesis as well as post-translational regulation (Guo and Gan 2005). Transcriptome analyses in Arabidopsis thaliana revealed that among 2,491 genes expressed during developmentally controlled senescence, approximately 800 genes are specifically up-regulated 
(Buchanan-Wollaston et al. 2003; Guo and Gan 2005). Despite a remarkable difference in the induced gene expression patterns observed between developmentally induced and dark-induced senescence, approximately 500 genes representing various functional categories, are common to the two forms of triggered leaf senescence (Buchanan-Wollaston et al. 2005; van der Graaff et al. 2006). Fifty-nine genes encoding transcription factors from 20 different gene families were shown to be induced both in developmental- and darkinduced senescence (Buchanan-Wollaston et al. 2005). The two largest groups of senescence-related transcription factors are the NAC and WRKY superfamilies. In Arabidopsis, the NAC domain protein family consists of 109 members, many of which are implicated in different developmental and stress response processes, and more than one-fifth of these genes are specifically induced during developmentally triggered senescence (Buchanan-Wollaston et al. 2005; Olsen et al. 2005). AtNAP, coding for a NAC family transcription factor, is highly expressed in senescing leaves and loss-of-function mutants of this gene display significantly delayed leaf senescence (Guo et al. 2004; Guo and Gan 2006).

At WRKY proteins, comprising 74 members, constitute the second largest group of transcription factors in the senescence transcriptome (Guo et al. 2004). WRKY proteins contain one or two domains of a conserved peptide stretch of about 60 amino acids, designated the WRKY domain, encompassing a Zn-finger motif (Ülker and Somssich 2004). The WRKY domain shows high binding affinity to a distinct cis-acting DNA element termed the W Box (T/CTGACC/T), although altered binding preferences have also been observed (Ülker and Somssich 2004). Specific WRKY family members show altered expression in various tissues and organs as well as in response to a wide range of pathogens, pathogenmimicking stimuli and different abiotic stresses suggesting a role of WRKY factors both in plant defense and plant developmental programs (Ülker and Somssich 2004). AtWRKY6 was shown to be strongly up-regulated during the progression of leaf senescence and also by treatments with salicylic acid (SA), jasmonic acid (JA) and ethylene (ET; Robatzek and Somssich 2001). Analyses of AtWRKY6 target genes identified SenescenceInduced Receptor Kinase $(S I R K=F R K 1)$ encoding a signaling component also identified in the FLS2-dependent defense pathway (Ülker and Somssich 2004). Overexpression, RNAi or knock-out lines of another gene, AtWRKY53, showed accelerated and delayed senescence phenotypes, respectively (Miao et al. 2004). However, the potential functions of the majority of the leaf senescence-associated AtWRKY transcription factors remain to be elucidated.
Here we report the functional analysis of AtWRKY70 exhibiting high expression levels in senescing leaves. The developmental- and darkinduced leaf senescence phenotypes of two independent Atwrky70 T-DNA insertion lines were analyzed as was the role of $A t W R K Y 70$ on SA- and JA/ETdependent defense-related genes during leaf senescence. In addition, the expression of AtWRKY70 was studied in transgenic plants and mutants defective in SA- and JA/ET-mediated signal transduction pathways.

\section{Materials and methods}

\section{Plant material and growth conditions}

Arabidopsis seeds were sown in plastic pots (diameters $8 \times 8 \times 8.5 \mathrm{~cm}$ ) with Minitray soil (Gebr. Patzer, Sinntal-Jossa, Germany), incubated for 3 days at $2-5^{\circ} \mathrm{C}$, subsequently transferred to a phytochamber (Heraeus Voetsch, Balingen, Germany) and grown under $12 \mathrm{~h}$ light $/ 12 \mathrm{~h}$ dark photoperiod and PFD of $80 \mathrm{mmol}$ photons $\mathrm{m}^{-2} \mathrm{~s}^{-1}$ at $22^{\circ} \mathrm{C}$ and $60 \%$ relative humidity. The mutants cpr5 (N3770), npr1 (N3726), jar1 (N8072), ein2 (N3071), ctr1 (N8057) were obtained from the Nottingham Arabidopsis Stock Centre. The mutant aos (knock-out line \#1,180 from the T. Jack T-DNA lines collection, Columbia-6 background) was supplied by Ken Feldmann (Ceres Inc., Malibu, CA, USA), $n d r 1$, edr1, and transgenic line expressing $N a h G$ gene were obtained from Paul Schulze-Lefert (Max-Planck Institute for Plant Breeding Research, Cologne, Germany), and pad4 was kindly provided by Jane Parker (Max-Planck Institute for Plant Breeding Research, Cologne, Germany). All plants were derived from the Columbia-0 ecotype unless mentioned. The Atwrky70-1 insertion mutant SALK 025198 was obtained from the Arabidopsis Resource Center whereas Atwrky70-2 corresponds to GABI-Kat line 752F08 (Rosso et al. 2003).

Construction of plasmids and generation of transgenic lines

DNA sequences of $1.9 \mathrm{~kb}$ AtWRKY70 upstream regulatory region, and the first $87 \mathrm{bp}$ of exon I were amplified from Columbia-0 genomic DNA using primers AtWRKY70-Pr-F: 5' (GWF) tacacaaccactaaactttacggc3' and AtWRKY70-Pr-R: 5' (GWR) cgaggagttgctgaagctg agttgt $3^{\prime}$, and cloned into $p D O N R 207$ Gateway ${ }^{\circledR}$ vector (Invitrogen). The Entry clone was recombined into the Gateway ${ }^{\circledR}$-compatible binary expression vector 
pJawohl11 (a derivative of pBIN19) to generate a translational promoter-GUS fusion construct. To generate $P_{\text {AtWRKY70 }}: G U S$ transgenic lines, Arabidopsis Col-0 plants were transformed via Agrobacterium tumefaciens strain GV3101 carrying the pMP90RK plasmid (Koncz and Schell 1986) and the appropriate construct using the inflorescence dipping method of Clough and Bent (1998). The histochemical assay to monitor for GUS activity in $P_{A t W R K Y 70:: G U S \text { transgenic }}$ plants was carried out according to the method described by Jefferson et al. (1987).

Full length AtWRKY70 cDNA (sequence deposited in Genbank under the accession number AF421157) was generated by RT-PCR starting from RNA derived from floral tissue of Columbia-0 ecotype and amplification of the cDNA with the primers AtWRKY70-F: $5^{\prime}(G W F)$ taatggatactaataaagcaaaaaagc $3^{\prime}$ and AtWRKY70-R: 5' (GWR) cagatagattcgaacatga actgaag $3^{\prime}$. The cDNA was cloned into the $p D O N R 201$ Gateway ${ }^{\circledR}$ vector (Invitrogen). The AtWRKY70-GFP expression construct was obtained by recombining the pENTR201 AtWRKY70 clone into the Gateway ${ }^{\circledR}$ compatible binary vector $p A M-K a n$ 2x35S::GW-GFP, a pPAM (GenBank accession AY027531) derivative.

The universal Gateway-compatible extensions for the $\mathrm{BP}$ recombination reactions (between an attBflanked PCR product and a donor vector containing $a t t \mathrm{P}$ sites to create an entry clone) were: $G W F$ (attB1) 5'ggggacaagtttgtacaaaaaagcaggctta3', GWR (attB2) 5'gg ggaccactttgtacaagaaagctgggtc $3^{\prime}$.

DNA sequences were determined by the DNA core facility (ADIS) of Max-Planck Institute for Plant Breeding Research on Abi Prism 377, 3100 or 3730 sequencers (Applied Biosystems, Weiterstadt, Germany) using BigDye-terminator v3.1 chemistry. Premixed reagents were from Applied Biosystems.

Subcellular localization studies

Peels of onion (Allium cepa L.) epidermal cell layers were placed inside up on Murashige and Skoog (MS) plates. The expression construct $p A M-K a n$ 2x35S::AtWRKY70-GFP and control construct $p A M$ Kan $2 x 35 S:: G F P-G F P$ (0.6 mg of DNA) were introduced into onion cells using a pneumatic particle gun (Biorad). The conditions for bombardments were: vacuum of $28 \mathrm{in}$. $\mathrm{Hg}$, helium pressure of 1,100 or 1,300 psi, and $6 \mathrm{~cm}$ of target distance using gold microcarriers. After bombardment, the peels were incubated on the MS plates for $24 \mathrm{~h}$ at $22^{\circ} \mathrm{C}$. Samples were transferred to glass slides and observed under a fluorescence microscope (Leica MZ12 with Mercury HBO 50 W/Ac lamp and FITC filter).
RNA extraction and cDNA synthesis

Total RNA was isolated from $100 \mathrm{mg}$ of fresh, healthy Arabidopsis leaf tissue. The tissue was flash-frozen and ground in liquid nitrogen. Total RNA was extracted with $1 \mathrm{ml}$ RNAwiz extraction reagent (Ambion, Huntingdon, Cambridgeshire, UK) following the supplier's protocol. DNase I treatment was performed using the DNA-free reagent (Ambion) for $20 \mathrm{~min}$ at $37^{\circ} \mathrm{C}$, and reaction composition was as suggested by the producer. First strand cDNA was synthesized using $2.5 \mu \mathrm{g}$ of RNA, SuperScript II RNase (Invitrogen) and Oligo dT 19 RT (Ambion) following manufacturers' instructions.

\section{RT-PCR, RNA-blot analyses and DNA labeling}

For RT-PCR analyses, $2 \mu \mathrm{l}$ of the first strand cDNA was used as template and reactions were standardized using primers specific to Arabidopsis tubulin $\beta$-subunit (At5g44340) (F: 5'acgtatcgatgtctattcaacga3' and R: 5'at atcgtagagagcctcattgtcc $\left.3^{\prime}\right)$. Subsequent PCRs were performed as indicated in the figures for various numbers of cycles under the following conditions: $94^{\circ} \mathrm{C}$ for $15 \mathrm{~s}$, $60^{\circ} \mathrm{C}$ for $30 \mathrm{~s}$, and $72^{\circ} \mathrm{C}$ for $1 \mathrm{~min}$, followed by a final extension at $72^{\circ} \mathrm{C}$ for $10 \mathrm{~min}$ using the following primer sets: PR1 (At2g14610), F: 5'tcgtctttgtagctcttgtaggtg3' and R: 5'ttcattagtatggcttctcgttca3'; PR2 (At3g57260), F: $5^{\prime}$ ccaccaatgttgatgattcttct $3^{\prime}$ and R: 5'atactcatccctgaaccttc ctt3'; PR3 (At3g12500), F: 5'tcgaatttgatttcacactgttc3' and $\mathrm{R}: 5^{\prime}$ ctgtagtttcatgggaagtctggc3'; PDF1.2 (At5g44420), F: $5^{\prime}$ taatcatcatggctaagtttgctt3' and R: 5'atacacacgatttagcac caaaga3'; COR1 (At1g19670), F: 5'gttacattcttgtagccccac ag3' and R: 5'ttctttgcccattcttacacat3'; SEN1 (At4g35770), F: 5'ccactgcttttaacacaacatca3' and R: 5'agcagtgagaagat cagttgagg3'; SEN2 (At1g20620), F: 5'ccagtctccaacaacatctc ttc3' and R: 5' atatcctcatggtttcaggact3'; SEN4 (At4g30270), F: 5'ttcttcttcacgactcttctcgt $3^{\prime}$ and R: 5' gtggaaacctcctatggtc agta3'; SRG1 (At1g17020), F: 5'gttgagaaactcgacttcgc3' and R: 5' gatcttagctacgctctgcac3'; SRG2 (At3g60140), F: 5' gacagctgcctcggcgtttcag3' and R: 5' gttaccttgatcataacccgc3', SAG29 (At5g13170), F: 5' gatcaatcaccatttcctcgc3' and R: 5'acgaatcccaccacgtttgg 3'; SAG12 (At5G45890), F: 5' gta ccaaaacgtttcttctggtg $3^{\prime}$ and R: 5' ccatgaattcttgatgatccaat $3^{\prime}$; SAG24 (At1g26910), F: 5'ggaaagccataccctaaatcaag3' and R: 5'aggatatcaactccacatcgaca3'; CAB (At3g27690), F: 5'gtcaagtctactcctcagagc $3^{\prime}$ and R: 5'cttccccaagagggaccacc tc3'; pectate lyase (At5G63180), F: 5' cgtctttgcccgttccgacc3' and R: 5'attgcatcaattaacccgtcg3'; EXGT (At4G37800), F: $5^{\prime}$ acttgttcggccgtgtgagc $3^{\prime}$ and R: 5'caatcatcggcttcccataacg 3'; GST (At5G17220), F: 5'tctctctacttcaataaatctccacc3' and R: 5'gagtttatgagaattgggcaattag3'; SIRK (At2g19190), F: 5'cat cgatttattcacaagctttgc 3' and R: 5'ttctgaactcactatacgcggtgtc 3'; RPS17 (At1g79850), F: 5'ctctctctaagcccaattccttc3' and 
R: 5'tcagattgaaaacacactcatgg3'; UBP8 (At5g22030), F: 5' taccagaggaggagacttattgg $3^{\prime}$ and R: 5'tcaacattcagcttct atcgag3'.

Northern blotting and radioactive labeling of the probe was performed using standard molecular procedures and random prime labeling. The AtWRKY70 cDNA probe was PCR-amplified using the WK70-F and $W K 70-R$ primers. Wherever possible, the primers used for RT-PCR were chosen such that DNA contaminations of the cDNA samples would yield fragments of a larger size due to the presence of intron sequences.

\section{Dark-induced leaf senescence}

Leaves or entire aerial parts of 2-week-old Arabidopsis plants were excised (seedlings were excised by cutting the hypocotyl approximately $5 \mathrm{~mm}$ below the cotyledons) and placed into microtiter plates filled with sterile water allowing good water contact of leaf pedicels and hypocotyls of seedling. The plates were kept in darkness at $22^{\circ} \mathrm{C}$ for $3-4$ days. For intact plant senescence experiments, 2 -week-old soil grown plants were transferred from the growth room to a container covered with a black box and placed in a dark room for 5-8 days.

\section{Results}

AtWRKY70 is up-regulated during developmentally induced leaf senescence

We took advantage of publicly available microarray data to gain information on the temporal and spatial expression patterns of AtWRKY70 (At3g56400) in specific organs at various developmental stages during the entire life cycle of Arabidopsis (Zimmermann et al. 2004; Schmid et al. 2005). Expression of AtWRKY70 was not detected in the embryo, in meristematic tissue, in roots, flower buds, petals, carpels, stamen, pollen grains or siliques. However, AtWRKY70 was constitutively expressed during all leaf development stages with highest signal intensity found in senescing leaves. Additionally, AtWRKY70 was also found to be expressed in floral abscission zones and flower sepals.

To substantiate and to extend on the expression data, we generated AtWRKY70 promoter GUS reporter lines $\left(P_{A t W R K Y 70}: \because G U S\right)$ expressing a translational fusion construct comprising $1.9 \mathrm{~kb}$ of AtWRKY70 upstream regulatory region, the first $87 \mathrm{bp}$ of $A t W R K Y 70$ exon I and the GUS reporter gene. Tissue-specific AtWRKY70 expression at different developmental stages was determined in 3-day-old etiolated seedlings, 15-day-old light-grown seedlings, 30-day-old plants, and in leaves and inflorescences from soil-grown 42-day-old mature plants. Consistent with the microarray data, GUS activity staining revealed a continuous increase in GUS activity staining during progression of leaf development. The AtWRKY70 gene was expressed at high levels throughout most stages of leaf growth but increases gradually up to the point of leaf senescence (Fig. 1a, panel 1). Moreover, GUS activity staining was also detected in various floral organs such as stigmatic papillae and the flower abscission zone (Fig. 1a, panels 2-5). However, at later stages of flower development, GUS activity staining was no longer detected in stigmatic papillae, whereas it remained persistent in the abscission zone (Fig. 1a, panel 2). Thus, AtWRKY70 expression patterns suggest a possible function during senescence of leaves and flowers but also for a need of $A t$ WRKY70 at earlier stages of leaf development.

\section{AtWRKY70 targets to the nucleus}

The $A t$ WRKY70 protein is predicted with high probability to be targeted to the nucleus (PENCE PA-SUB, $100 \%$; PSORT, 98\%). To test this prediction, we transiently transfected an expression construct, $2 x 35 S:$ : AtWRKY70-GFP, encoding a full-length AtWRKY70 protein fused to GFP into onion (Allium cepa L.) epidermal cells. As a control, a construct expressing the GFP reporter (2x35S::GFP) was included. Microscopic analysis revealed that at all time points tested $(6,10$, and $24 \mathrm{~h}$ post transfection) AtWRKY70-GFP was solely visualized in the nucleus confirming that AtWRKY70, like other WRKY family members, resides almost exclusively within the nucleus (Fig. 1b). In contrast, $2 x 35 S:$ GFP expression resulted in detection of the protein throughout the cells.

\section{Analysis of two Atwrky70 T-DNA insertion lines}

To gain deeper insight into the function of AtWRKY70 in planta, we obtained two independent insertion mutants, Atwrky70-1 (SALK_025198) and Atwrky70-2 (GABI_752F08), with T-DNAs located within the first intron and third exon of the AtWRKY70 gene, respectively (Fig. $2 \mathrm{a}$ ). In both cases, the presence of the $\mathrm{T}$ DNA was confirmed by PCR amplification of specific bands using primer pairs derived from the AtWRKY70 gene and the left border of the T-DNA. Homozygous Atwrky70 knock out lines were isolated based on the absence of AtWRKY70 gene specific PCR products (Fig. 2b). Moreover, both homozygous Atwrky70 insertion lines showed no detectable AtWRKY70 transcript 
a

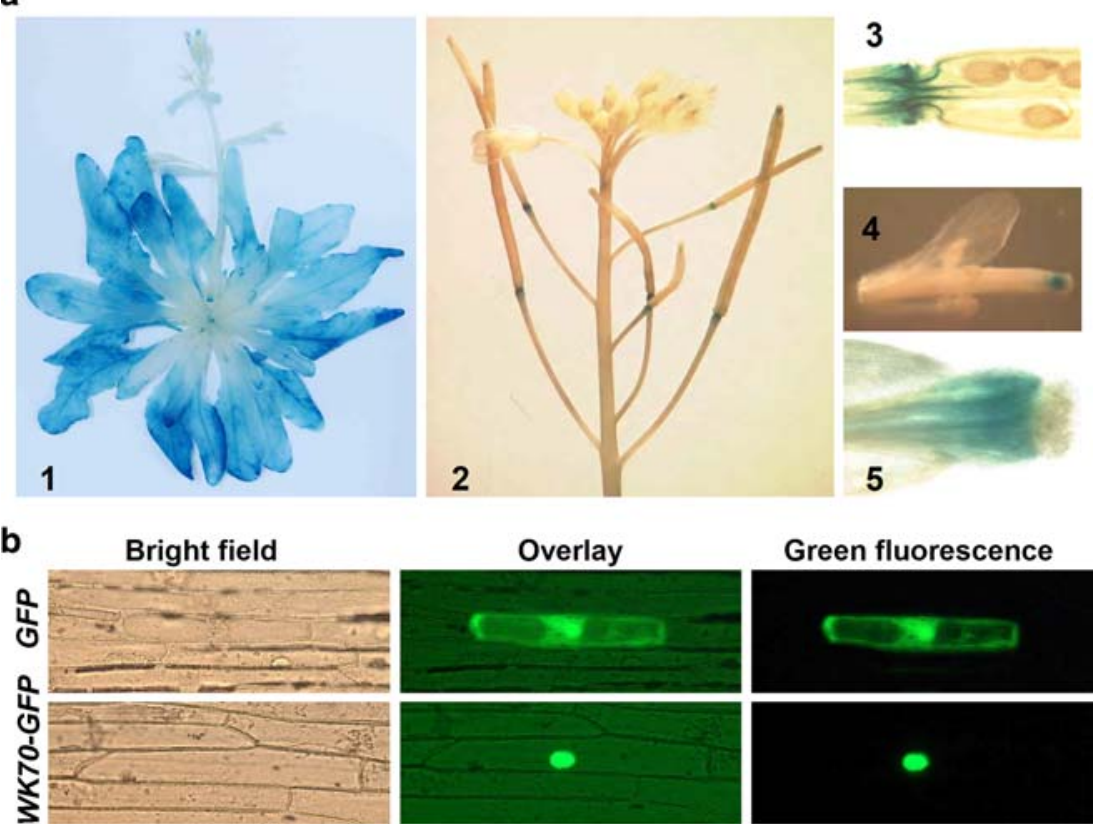

Fig. 1 a Expression analysis of a representative $P_{A t W R K Y 70}: G U S$ transgenic plant. Part 1 GUS-activity staining in a 42 -day-old plant; part 2 GUS-activity staining in total inflorescences; part 3 close-up view of GUS-activity staining detected within a floral organ abscission zone; parts 4 and 5 GUS-activity staining observed in the residual stigmatic papillae (close-up in e) at later stages of flower development. b Nuclear localization of AtWRKY70. Tran-

(Fig. 2c) and thus very likely represent complete lossof-function mutants. During the entire period of development, the two Atwrky70 knock-out mutants were slightly reduced in size compared to wild-type plants (Fig. 2d, and data not shown).

\section{Loss of AtWRKY70 function promotes}

both developmentally and dark-induced leaf senescence

We investigated the role of AtWRKY70 in leaf senescence by analyzing both the developmentally induced and dark-induced senescence programs. The Atwrky 70 insertion lines and wild-type plants were grown under standard greenhouse or phytochamber conditions to monitor natural senescence. Under the tested conditions, the Atwrky70 loss-of-function mutants showed markedly earlier senescence compared to wild-type plants at 6-7 weeks post germination. The wild-type plants displayed significant accumulation of red anthocyanin pigments but moderate leaf yellowing; both considered the hallmarks of the early leaf senescence stages. On the contrary, Atwrky70 mutant plants exhibited severe yellowing of leaves, resulting from intensified degradation of chlorophyll, which is indicative of an advanced leaf senescence phase (Fig. 3a). However, sient expression of $2 x 35 S:: A t W R K Y 70-G F P$ in onion epidermal cells. Bright field images of the respective onion epidermal cells are shown to the left. At WRKY70-GFP is visible solely in the nucleus (lower right), whereas the control GFP-GFP protein is present throughout the entire cell (top right). Middle column shows bright field image overlays with those obtained using a GFP filter

bolting and flowering times were nearly identical between the Atwrky70 mutants and wild-type plants revealing that the earlier appearance of leaf yellowing in Atwrky70 mutants plants was not the consequence of a generally accelerated ontogenesis, but rather occurred due to an earlier onset of the developmentally induced senescence program.

We next examined dark-induced senescence, a procedure that is commonly used to artificially trigger senescence (Weaver and Amasino 2001). Detached leaves or entire aerial parts of 2-week-old soil grown plants of the two independent Atwrky 70 insertion lines and wild-type plants were subjected to darkness for 3-4 days. Within this time period, leaves of the Atwrky70 mutants showed severe yellowing and cell death while leaves of wild-type plants only became pale green (Fig. 3b). Additionally, we also examined darkinduced leaf senescence of intact plants and observed that leaves of both Atwrky70 mutants senesced more rapidly than wild-type leaves when kept in the dark for 5-6 days (data not shown) again pointing towards a function of AtWRKY70 in leaf senescence.

Leaf senescence is accompanied by decreased expression of genes related to photosynthesis and protein synthesis and increased expression of senescence-associated genes (SAGs), with different leaf 

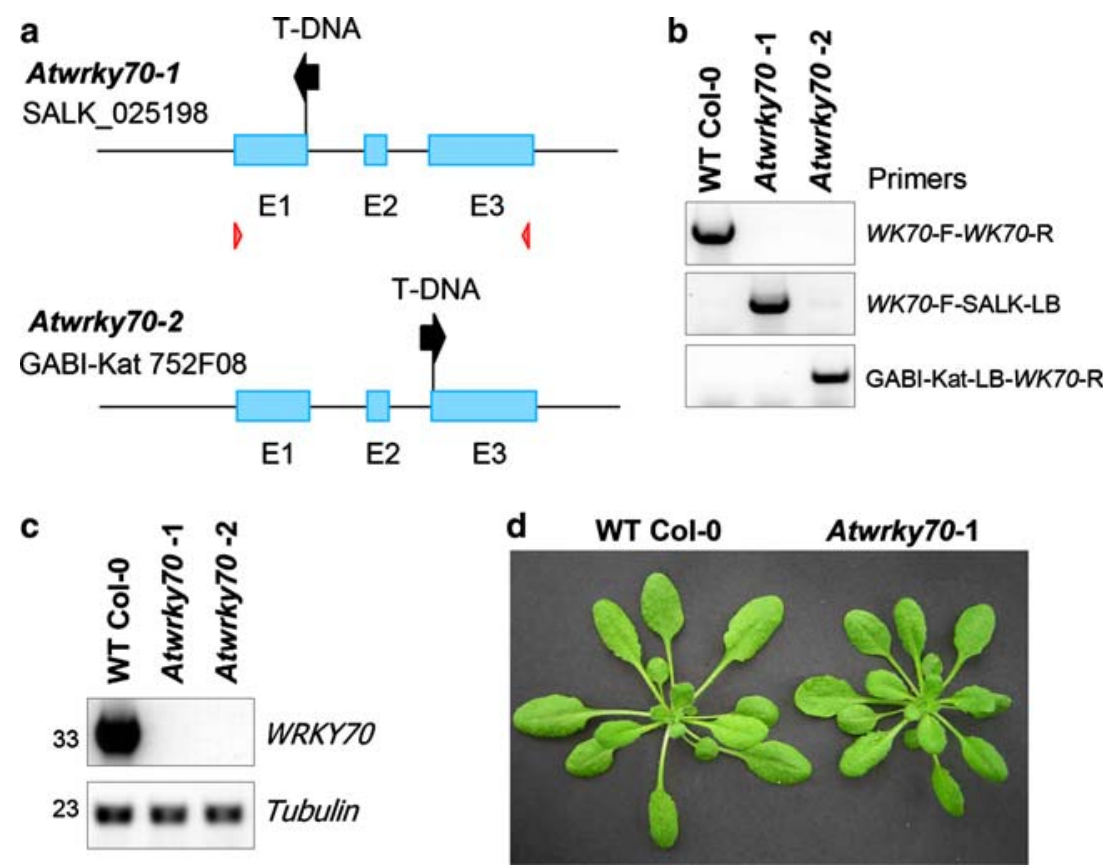

Fig. 2 Identification and characterization of homozygous Atwrky70 T-DNA insertion alleles. a Schematic representation of the T-DNA insertion sites within the AtWRKY70 locus. The three exons (marked E1,E2 and E3) are highlighted in blue. Positions of the T-DNA insertions are marked by black arrows with the arrowheads pointing in each case towards the left border site within the T-DNA. b Verification of homozygous Atwrky70 mutant lines. Gene-specific and respective T-DNA-specific primers were used to detect the presence of the wild-type and mutant AtWRKY70 alleles in genomic DNA from representative Atwrky70-1 and Atwrky70-2 homozygous plants, and from wildtype control. Origin of the genomic DNA is shown at the top

senescence stages classified based on the expression patterns of specific marker genes (Oh et al. 1997; Yoshida et al. 2001). To determine the effect of $A t$ WRKY70 on senescence signaling, we measured transcript levels of several senescence-associated genes in the Atwrky 70 mutant and wild-type plants using semi quantitative RT-PCR. These included genes related to oxidative stress such as SRG1 (oxidoreductase), GST (glutathione S-transferase) and SEN2 (catalase 3), genes related to cell wall degradation, breakdown of macromolecules and transport to other plant parts such as SRG2 (=DIN2; dark-induciable gene 2, glycosyl hydrolase), pectate lyase, EXGT (xyloglucan:xyloglucosyl transferase), and SEN4 (=MERI-5, endo-xyloglucan transferase), genes related to protein synthesis and degradation such as $S A G 12$ (senescence-specific cysteine proteinase), RPS17 (chloroplast ribosomal protein S17) and SAG24 (60S ribosomal protein L10), genes related to the progression of photosynthesis such as $C A B$ (chlorophyll $a / b$ binding protein), genes related to signaling such as $\operatorname{SIRK}$ (senescence-induced receptor-like kinase) and genes with unknown functions but whereas the primer combinations used for PCR analysis are given to the right. c The AtWRKY70 T-DNA insertion lines appear to be complete loss-of-function mutants. RT-PCR was performed with $A t W R K Y 70$ gene-specific primers and the respective DNA templates as indicated above each lane. AtWRKY70 transcript could not be detected in 30-day-old plants homozygous for the TDNA insertions but was clearly detected in wild-type Col-0 control plants. Expression of Tubulin was used as a loading control. Numbers of RT-PCR cycles performed are indicated to the left. d AtWRKY70 insertion lines are somewhat smaller than wild type plants. Shown is a comparison between WT and Atwrky70-1

shown to be associated with senescence such as $S A G 29$ (nodulin MtN3 family protein) and SEN1, an early indicator of natural and dark induced senescence.

Initially, we examined the expression of senescenceassociated genes assumed to be altered in 40-days-old plants showing early signs of senescence. At this stage, plants have bolted and some of the older rosette leaves showed yellowing on their edges. The Atwrky70-1 mutant plants had more of such yellowing leaves. As expected, the expression of $C A B$, pectate lyase, and $E X G T$ were down-regulated and the expression of $G S T, S R G 1, S R G 2$ and SIRK were up-regulated in the Atwrky70-1 mutant compared to the wild-type plants (Fig. 4a). The expression of SAG29 was slightly lower in Atwrky70-1 than in wild-type plants.

To determine if $A t$ WRKY70 plays a role in the initiation of senescence we monitored the expression of several genes, including some early senescence-associated marker genes, in 30-days-old Atwrky70-1 and Atwrky70-2 mutant and wild-type plants showing no visible signs of senescence. To exclude any bias in the selection of individual leaves for sampling and avoid 

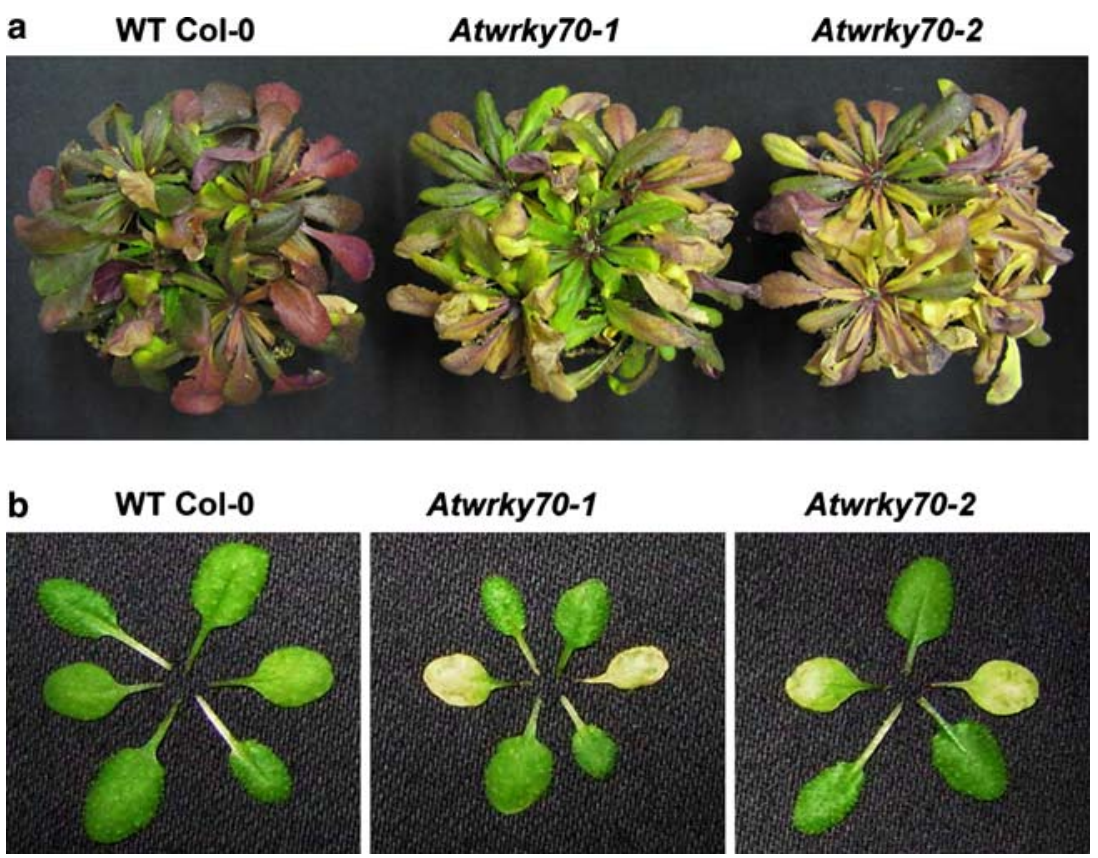

Atwrky70-1

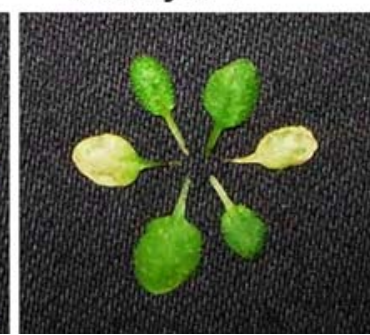

Atwrky70-2

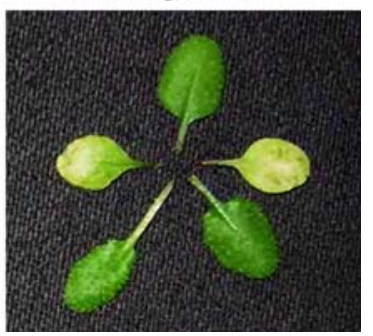

Fig. 3 Senescence phenotype of Atwrky70 mutant plants. a Progression of natural senescence in Atwrky70 mutants and wildtype plants grown under controlled conditions. The number of yellow and necrotic leaves is higher in 7-week-old Atwrky70-1 and Atwrky70-2 mutant plants compared to wild-type due to accelerated developmental senescence. Inflorescenses were removed for clarity of the senescence phenotype. b Dark-induced

expression differences resulting from wounding we chose to harvest the entire aerial parts of the plant. No differences in the expression of some late senescence-associated genes (pectate lyase, EXGT, SAG12, and $S A G 24$ ) were observed between wild-type plants and the Atwrky70-1 mutant (Fig. 4b), but in AtWRKY70-2 the expression of SAG12 was lower and that of pectate lyase was somewhat higher. Since expression of the pectate lyase gene is downregulated and that of $S A G 12$ is upregulated during progression of senescence (compare with Fig. 4a), one possible explanation for this observed difference was that the physiological states of the two mutants at sampling time were not completely identical. Alternatively or in addition, the two loss-of-function alleles may differ somewhat in their rates of leaf developmental progressions (Fig. 4b). The expression of SAG29 was again slightly lower in the Atwrky 70 mutants. On the other hand, higher transcript levels of SEN1, SEN2 and SRG1 were observed in the leaves of the Atwrky70 mutants compared to leaves of wild-type plants (Fig. 4b). No differences in expression were detected for SEN4. Together these data show that loss-of- $A t W R K Y 70$ function influences the expression levels of certain senescence-associated markers genes even at early stages of the leaf senescence program. senescence phenotype of Atwrky70 mutant plants. Aerial parts of 2-weeks-old wild type and Atwrky70 mutants plants grown under long day condtions in the phytochamber were excised by cutting their hypocotyls $5 \mathrm{~mm}$ below their cotyledons. The excised plantlets were transferred to microtiter wells containing water and kept under dark conditions for 3, 4 days. For clarity, the leaves were dissected from the seedlings before being photographed

AtWRKY70 represses both SA and JA/ET mediated defense marker genes expression

The regulatory network governing leaf senescence has substantial crosstalk with plant defense signaling pathways (Grbic and Bleecker 1995; Morris et al. 2000; He et al. 2002). In Arabidopsis, at least three genetically distinguishable defense pathways are characterized: the SA-, JA- and ET-mediated resistance pathways (Glazebrook 2005). The levels of these signaling molecules increase during senescence and can modulate the expression of specific downstream genes (Morris et al. 2000; Buchanan-Wollaston et al. 2005). SA also induces the expression of pathogenesis-related $(P R)$ genes encoding the acidic isoforms of proteins such as $P R 1, P R 2, P R 3$, and PR5 as well the transcriptional regulator NPR1 (non-expressor of PR1; Ward et al. 1991). Moreover, JA/ET activate the expression of plant defensin1.2 (PDF1.2), and coronatine resistant 1 (COR1; Benedetti et al. 1998; Glazebrook 2005). To determine the activation state of the SA, JA and ET pathways in the Atwrky70 mutants during senescence, the steady-state transcript levels of NPR1, PR1, PR2, PR3, PR5, COR1, and PDF1.2 were evaluated and compared to that of wild-type plants 30 days post germination. Basal expression levels of the SA-responsive 

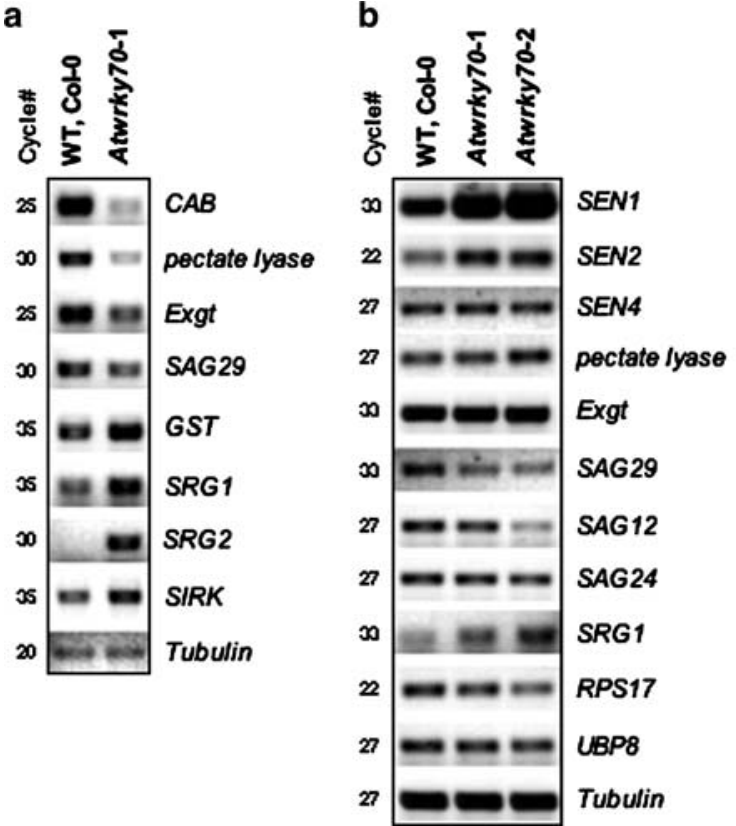

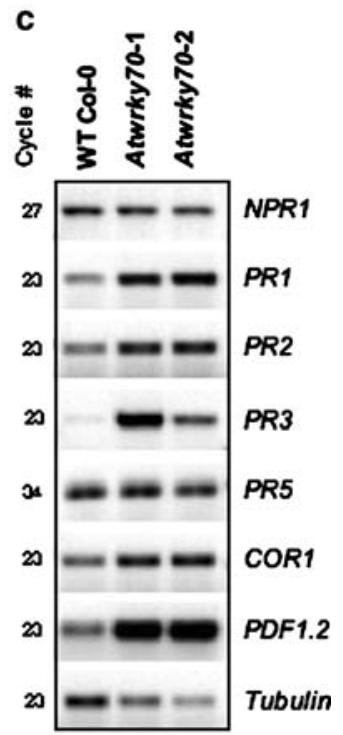

Fig. 4 a Early senescence symptoms in the Atwrky70-1 mutant correlate with transcriptional up-and down-regulation of genes associated with senescence. Semi-quantitative RT-PCR analyses of 40-days-old Col-0 wild-type and Atwrky70-1 mutant plants using senescence-related marker genes (indicated to the right). Number of RT-PCR cycles are given to the left. Ten plants were sampled by punching out $10 \mathrm{~mm}$ diameter leaf disks from the seventh oldest leaf, counting from the first true leaf, of each plant grown in the phytochamber. RNA was isolated from the pooled samples. $C A B$ chlorophyll $a, b$ binding protein coding gene, $E X G T$ xyloglucan:xyloglucosyl transferase, $S A G 29$ senescenceassociated gene 29, GST glutathione S-transferase gene, SRG1 senescence-related gene $1, S R G 2$ senescence-related gene 2 (=DIN2), SIRK senescence-induced receptor-like kinase. b Expression of genes associated with early senescence are elevated in Atwrky 70 mutant plants. RT-PCR analyses on 30-day-old Col-0 wild-type and Atwrky70 mutant plants using senescencerelated marker genes (indicated to the right). cDNA templates used in each reaction are labeled above each lane. Number of RTPCR cycles are given to the left. SEN1 senescence-associated gene

$P R 1$, and $P R 2$ genes, the JA/ET-responsive defense marker genes COR1 and PDF1.2, as well as that of $P R 3$ which responds to both stimuli, were significantly elevated in the Atwrky70 mutant plants when compared to wild-type (Fig. 4c). In contrast to this, no major changes were observed in the expression of NPR1 and PR5. The accelerated senescence of the Atwrky70 mutants may in part be the consequence of deregulated expression of some defense genes resulting in an elevated stressed-state of such plants.

To gain additional insight into the nature of the signals that are common to plant defense and the Atwrky70-mediated early senescence phenotype, we studied the expression patterns of AtWRKY70 in different mutants and transgenic plant lines affected in known host defense signal transduction pathways. The
1, SEN2 senescence-associated gene 2, SEN4 senescence-associated gene 4, EXGT xyloglucan:xyloglucosyl transferase, $S A G 29$ senescence-associated gene 29, SAG12 senescence-associated gene 12, SAG24 senescence-associated gene 24, SRG1 senescence-related gene $1, R P S 17$ chloroplast ribosomal protein coding gene, $S 17, U B P 8$ ubiquitin-specific protease coding gene 8 . c The basal expression levels of some pathogen-responsive marker genes are elevated in Atwrky70 mutant plants. Transcript levels of NPR1, PR1, PR2, PR3, PR5, COR1 and PDF1.2 in wild-type, Atwrky70-1 and Atwrky70-2 plants were detected by RT-PCR NPR1 non expressor of PR1, PR1 pathogenesis related 1, PR2 pathogenesis related 2, $P R 3$ pathogenesis related 3, $P R 5$ pathogenesis related 5, COR1 coronatine resistant 1, PDF1.2 plant defensin1.2. The number of RT-PCR cycles performed are given to the left. The expression levels of tubulin (At5g44340) and UBP8 (At5g22030) ubiquitin-specific protease eight were used as loading controls for $\mathbf{b}$ and $\mathbf{c}$. RNA derived from entire aerial plant organs was isolated from pooled samples of six plants each grown in a phytochamber

SA hyper-accumulating mutant cpr5 (constitutive $P R$ gene expressor 5 ) also showed early senescence in older leaves (Yoshida et al. 2002b). NahG transgenic plants failed to accumulate SA, whereas pad4 (phytoalexindeficient 4) and nprl (non-expressor of PR 1) were defective in SA signaling. All of these SA mutants exhibited a delayed senescence phenotype (Morris et al. 2000). It is known that JA application induces premature senescence and that endogenous jasmonate levels increase fourfold to fivefold during plant senescence (He et al. 2002). The aos (allene oxide synthase) and jar1 (jasmonic acid resistant 1) mutants are impaired in JA biosynthesis or its activation, respectively (Park et al. 2002; Glazebrook 2005). The ETdependent pathway mutant ein2 (ethylene insensitive 2) also shows an early senescence phenotype (Oh et al. 
1997). The basal AtWRKY70 transcript levels were unaltered in the aos, jar1 and ein 2 mutants indicating that both JA- and ET-dependent signaling are not required for AtWRKY70 expression during developmental senescence. Contrastingly, AtWRKY70 transcript levels were slightly reduced in the cpr5 mutant, more strongly reduced in nprl and pad4 and completely abolished in NahG plants compared to wildtype at 40 days post germination (Fig. 5a). Thus, functional SA signaling pathway is required for
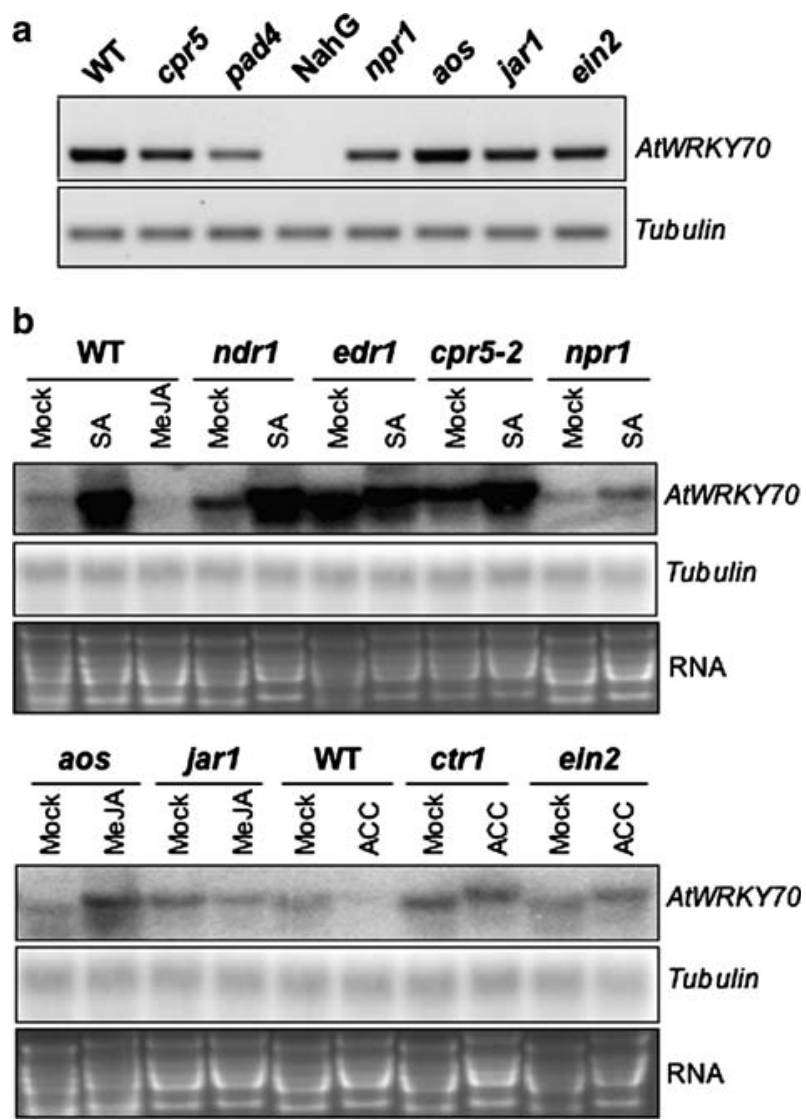

Fig. 5 a Endogenous $A t W R K Y 70$ transcript levels in various defense signaling mutants. Comparative basal AtWRKY70 transcript levels observed in the indicated Arabidopsis mutants impaired in different defense signaling pathways. cpr5, pad4, NahG and nprl define the SA dependent pathway, aos and jar1 the JA-dependent pathway and ctr1 and ein2 the ET-dependent pathway. PCR on genomic DNA showed no DNA contamination (data not shown). Tubulin was used as loading control. b Expression of AtWRKY70 in various defense signaling mutants upon different treatments. RNA samples were prepared from leaves of 22-day-old wild-type or mutant plants as indicated. After electrophoretic separation on the gel and blotting onto nylon membranes, the blot was probed with an AtWRKY70 specific DNA fragment. A tubulin probe and ethidium bromide stained ribosomal RNA bands were used to monitor equal RNA loading of each lane. Wild-type and mutant plants are indicated at the top and underlined, and the respective treatments are given for each lane. $S A$ salicylic acid, MeJA methyl jasmonate, $A C C$ 1-aminocyclopropane-1-carboxylic acid
AtWRKY70 expression during leaf senescence. Since overexpression or antisense suppression of AtWRKY70 caused no major alterations in endogenous levels of free SA, JA and ET in Arabidopsis leaves ( $\mathrm{Li}$ et al. 2004), the Atwrky70-mediated early senescence phenotype observed here was not a consequence of changes in the balance of these hormones.

We next analyzed AtWRKY70 transcript levels in 22-days-old mutant plants impaired in components of various defense signaling pathways upon specific treatments. Moreover, we expanded the array of mutants by including $n d r 1$ (non-race-specific disease resistance 1), compromised in the activation of CC-NB-LRR class of resistance proteins, edr1 (enhanced disease resistance 1), conferring elevated resistance to powdery mildew disease and ctr1 (constitutive triple response1) exhibiting constitutive activation of the ethylene signaling pathway (Kieber et al. 1993; Century et al. 1995; Frye et al. 2001). The mutants $n d r 1$, edr1, cpr5 and nprl were treated with SA, aos and jarl were treated with methyl jasmonate (MeJA) and ctrl and ein2 were treated with the natural precursor of ET 1-aminocyclopropane-1carboxylic acid (ACC). The endogenous basal and stimulus-dependent transcript levels of AtWRKY70 were not significantly affected by a $4 \mathrm{~h}$ treatment with MeJA or ACC either in wild-type plants or in the mutants impaired in JA/ET signaling strongly suggesting that the JA/ET signaling transduction pathways are not required for AtWRKY70 expression (Fig. 5b). Note that the slight reduction of AtWRKY70 transcript levels in ACC-treated wild-type plants was not consistently reproducible. In contrast to MeJA or ACC treatments, AtWRKY70 displayed a strong increase in expression upon treatment with SA. Basal expression of AtWRKY 70 was elevated in $n d r 1$ and cpr 5 mutants, but could be induced to higher levels upon SA application supporting the hypothesis that SA signaling positively affects AtWRKY70 expression (Fig. 5b). In contrast, high constitutive levels of AtWRKY70 transcript were observed in the edr1 mutant with no significant changes detected upon SA treatment. The reduction in AtWRKY70 transcript levels in the npr1 mutant before or after SA treatment (Fig. 5b) is consistent with previous reports ( $\mathrm{Li}$ et al. 2004). Collectively, our results support the role of AtWRKY70 as a senescence-associated gene and indicate a functional requirement of SA for its normal expression.

\section{Discussion}

Over the past decade our knowledge on the molecular events occurring during leaf senescence has expanded 
significantly (Buchanan-Wollaston et al. 2003). Moreover, global expression data revealed a common subset of genes, many encoding transcription factors, that are expressed both during developmentally and artificially induced senescence (Lin and Wu 2004; BuchananWollaston et al. 2005; van der Graaff et al. 2006). These findings were key steps forward in identifying potential transcriptional regulators modulating signal transduction pathways during the onset and progression of senescence. Our data on AtWRKY70 adds additional evidence that this individual transcription factor plays an important regulatory role in both of these processes and possibly also in the abscission of floral organs.

Leaf senescence can also be induced by several biotic and abiotic stresses (Buchanan-Wollaston et al. 2003) for which global transcript profiling data is available (Zimmermann et al. 2005). Heat, darkness and UV-B radiation are potent inducers of premature senescence that cause loss of chlorophyll and an increase in lipid damage, mimicking natural senescence and resulting in the regulation of senescence-associated genes (John et al. 2001; Swidzinski et al. 2002). AtWRKY70 is strongly down-regulated by these treatments which is consistent with Atwrky 70 mutants' early senescence phenotypes. In contrast, cycloheximide (CHX), an inhibitor of RNA-directed protein synthesis and senescence progression (Bieleski and Reid 1992) strongly induces AtWRKY7O expression levels. This strong activation of AtWRKY70 in the presence of $\mathrm{CHX}$ also indicates that this gene is very likely under negative regulatory control by a factor with a short half-life.

The concomitant changes in the transcriptional activities of several genes, especially the up-regulation of genes associated with early senescence such as SEN1, SEN2 and SRG1 in the Atwrky70 mutants, lends further support for a role of AtWRKY70 in partly regulating critical steps within the process of leaf senescence. SEN1 is known to be up-regulated in leaves during the initial stages of dark-, ethylene-, or ABA-induced senescence (Oh et al. 1996). SEN1, SEN2, SEN4 and $S R G 1$ contain numerous W box elements, the cognate binding sites for WRKY transcription factors. Thus, AtWRKY70 may play a role in the regulation of these genes either directly or by activating other WRKY genes. In fact, the SEN1 promoter has eight W box motifs (TGACC/T or C/TTGAC) five of which are extended $\mathrm{W}$ box elements (C/TTGACC/T). A similar situation is found in the promoters of $S E N 2,(7 \mathrm{~W}$ boxes, 2 of which are extended $\mathrm{W}$ box elements), of SEN4 (6 W box motifs of which 3 are extended W boxes), and of SRG1 (11 W boxes of which 3 are extended W-box elements). Except for SEN4, all of these genes were up-regulated in the Atwrky70 mutant plants.

Since senescence-associated genes are shown to accumulate in plant tissues not only during various phases of senescence but also during another form of PCD termed the hypersensitive response (HR; an active defense strategy against numerous pathogens), a significant overlap in gene transcription between these two physiological processes likely exists (Maleck et al. 2000; Quirino et al. 2000; Schenk et al. 2005). Indeed, AtWRKY70 expression is also altered in response to various pathogens including the hemibiotrophic bacterium Pseudomonas syringae pv tomato and the necrotrophic fungi Alternaria brassicicola and Botrytis cinerea (Li et al. 2004; Zimmermann et al. 2004; AbuQamar et al. 2006). Overexpression of AtWRKY70 caused heightened resistance to $P$. syringae and Erysiphe chichoracearum and enhanced susceptibility to $A$. brassicicola ( $\mathrm{Li}$ et al. 2004, 2006). In contrast, loss-ofAtWRKY70 function resulted in enhanced susceptibility to E. cichoracearum and B. cinerea (AbuQamar et al. 2006). Moreover, recent studies showed that necrotrophs deliberately trigger HR in the host, which subsequently leads to cell death and necrosis and hence might be a strategy used by these pathogens to initiate colonization of host tissue (Govrin and Levine 2000; La Camera et al. 2005). The fact that AtWRKY70 expression is regulated both by defense- and senescence-associated signals acting in a coordinated manner indicates that AtWRKY70 function may be required to integrate signals that link both pathways.

Treatment with SA appears to be a very potent inducer of AtWRKY70 expression and increases its transcript levels almost 30 -fold within $2 \mathrm{~h}$ of application (Fig. 5b; Li et al. 2004; Ülker and Somssich 2004; Zimmermann et al. 2005). Recently, SA was proposed to promote the expression of senescence-related genes solely in naturally occurring senescing leaves (Morris et al. 2000; Buchanan-Wollaston et al. 2005; van der Graaff et al. 2006). SA appears to be a signal required for AtWRKY70 expression in both young and senescing leaves, as no endogenous transcript can be found in SA-deprived $N a h G$ transgenic plants, and a strong reduction is observed in SA defective signal transduction mutants eds1, pad4 and nprl (Fig. $5 \mathrm{~b}$; Li et al. 2004; Zimmermann et al. 2005; Bartsch et al. 2006). Conversely, increased basal transcript levels of AtWRKY70 in SA hyper-accumulating mutants edr1, cpr5 and acd11 (accelerated cell death 11), at an early stage of plant development suggest that SA promotes AtWRKY70 expression (Fig. 5b; Frye et al. 2001; Brodersen et al. 2002; Yoshida et al. 2002a). The high constitutive levels of AtWRKY70 transcript in the edr1 
mutant are intriguing. EDR1 encodes a Raf-like mitogen-activated protein kinase kinase kinase (MAPKKK) that negatively regulates disease resistance (Frye et al. 2001). edr1 plants are not altered in their timing of senescence compared to wild-type plants. However, edr1 mutants display an enhanced ethyleneinduced senescence response, a phenotype that can be phenocopied by overexpressing a dominant negative variant of EDR1 (Frye et al. 2001; Tang and Innes 2002). It is therefore possible that AtWRKY70 contributes to this enhanced response by regulating/or interacting with an ethylene-dependent component. Endogenous AtWRKY70 expression kinetics varied in cpr5 mutant depending upon the developmental stage of the plants. At the early age (e.g., 22 days), AtWRKY70 expression is induced, while in senescing leaves a slight repression relative to wild-type plants was observed. The biological significance of these findings is currently unknown.

Our data suggest that AtWRKY70 can act as a repressor of some SA- and JA-response genes such as $P R 1, P R 2, P R 3, C O R 1$ and PDF1.2. This is partly in agreement to the data of AbuQamar et al. (2006) who also showed that in Atwrky70 mutant plants basal expression of the SA-dependent $P R 1$ is elevated. This finding, however, contradicts results obtained by $\mathrm{Li}$ et al. (2004), who found no impairment of $P R 1$ expression in an AtWRKY70 antisense line. Unfortunately, comparison of $P R 1$ expression of this antisense line with the Atwrky70 mutants recently analyzed by $\mathrm{Li}$ et al. (2006) was not reported. Thus, it remains unclear whether this observed difference is due to variations of the experimental set-ups used or due to off-target effects of the silencing construct used, since the antisense line is phenotypically not identical to the Atwrky70 mutant plants (Li et al. 2006). Still, several defense-related genes are known to be induced during leaf senescence, including $P R 1, P R 2, P R 3$ and $P D F 1.2$ (Wyatt et al. 1991; Hanfrey et al. 1996; Morris et al. 2000; Quirino et al. 2000; He et al. 2002), in a phenomenon known as Age-Related Resistance (Kus et al. 2002). Expression of $P R$ genes during senescence is probably linked to a protective function in aging leaves against invasion by opportunistic pathogens, thereby maintaining viability of the leaf until mobilization is complete and preventing infection of the rest of the plant (Kus et al. 2002).

In contrast to our study, AbuQamar et al. (2006) did not observe elevated basal expression levels of $P D F 1.2$ in Atwrky70 mutants although a difference in the temporal expression kinetics of PDF1.2 following pathogen challenge was seen in such mutants compared to wild-type plants. Atwrky70 mutants showed a clearly accelerated expression of PDF1.2 transcript in Botrytis-inoculated plants (maximum levels reached at $36 \mathrm{~h}$ $\mathrm{pi}$ in the mutant compared to $60 \mathrm{~h}$ in wild-type plants). Basal expression of PDF1.2 was not reported for the AtWRKY70 antisense line but was slightly enhanced in the Atwrky70-1 mutant (Li et al. 2006) consistent with our observations.

Finally, the increased basal AtWRKY70 transcript levels in the coil mutant reported recently $(\mathrm{Li}$ et al. 2004; AbuQamar et al. 2006) stand partially in disagreement with our findings revealing that AtWRKY70 expression is not significantly altered in two JA-signaling mutants aos and jarl (Fig. 5b) and with microarray data showing that AtWRKY70 expression was not altered in the mid-flowering stage of coil mutant plants (Buchanan-Wollaston et al. 2005). Again, these discrepancies may be due to the experimental differences or alternatively, specific to the coil mutant and thus not a general JA-related effect.

In spite of the biological and practical importance of leaf senescence, elucidation of the genes that control senescence has been complicated by the complexity of signaling pathways that appear to be involved. Due to an apparent functional redundancy within the signaling networks the task of defining the specific contribution of individual pathways to the senescence program is difficult and may explain why forward screens have only identified a very limited number of mutants directly associated with a senescence phenotype (Buchanan-Wollaston et al. 2005). It is for this reason that the analysis of potential transcriptional regulators remains an important area within this field of research. Here we have identified one transcriptional regulator, which signals through the SA-mediated pathway and thereby regulates a subset of genes common to senescence and plant defense. Identifying specific $A t$ WRKY70 in vivo target genes will be the next step required to gain a more in-depth knowledge of its role within these pathways and to more precisely define specific convergence points. Thus, elucidating the senescence mechanisms at the molecular level will remain a challenge but should yield valuable information not only on the regulation of developmental cell death, but also provide the necessary tools to prudently manipulate the senescence process in crops for increased plant longevity, productivity, stress tolerance, pre- or post-harvest storage and shelf life.

Acknowledgment We thank Dr. Karolina Pajerowska-Mukhtar for critically reading of the manuscript. M.S.M was supported by International Max Planck Research School (Max Planck Society, Munich, Germany). Financial support of B.U. was partly provided by the EU-funded REGIA project. 


\section{References}

AbuQamar S, Chen X, Dhawan R, Bluhm B, Salmeron J, Lam S, Dietrich RA, Mengiste T (2006) Expression profiling and mutant analysis reveals complex regulatory networks involved in Arabidopsis response to Botrytis infection. Plant $\mathrm{J}$ 48:28-44

Bartsch M, Gobbato E, Bednarek P, Debey S, Schultze JL, Bautor J, Parker JE (2006) Salicylic acid-independent enhanced disease susceptibility1 signaling in Arabidopsis immunity and cell death is regulated by the monooxygenase FMO1 and the nudix hydrolase NUDT7. Plant Cell 18:1038-1051

Benedetti CE, Costa CL, Turcinelli SR, Arruda P (1998) Differential expression of a novel gene in response to coronatine, methyl jasmonate, and wounding in the coil mutant of Arabidopsis. Plant Physiol 116:1037-1042

Bieleski RL, Reid MS (1992) Physiological changes accompanying senescence in the ephemeral daylily flower. Plant Physiol 98:1042-1049

Brodersen P, Petersen M, Pike HM, Olszak B, Skov S, Ødum N, Jørgensen LB, Brown RE, Mundy J (2002) Knockout of Arabidopsis accelerated-cell-death11 encoding a sphingosine transfer protein causes activation of programmed cell death and defense. Genes Dev 16:490-502

Buchanan-Wollaston V, Earl S, Harrison E, Mathas E, Navabpour S, Page T, Pink D (2003) The molecular analysis of leaf senescence-a genomics approach. Plant Biotechnol J $1: 3-22$

Buchanan-Wollaston V, Page T, Harrison E, Breeze E, Lim PO, Nam HG, Lin JF, Wu SH, Swidzinski J, Ishizaki K, Leaver CJ (2005) Comparative transcriptome analysis reveals significant differences in gene expression and signalling pathways between developmental and dark/starvation-induced senescence in Arabidopsis. Plant J 42:567-585

Century KS, Holub EB, Staskawicz BJ (1995) NDR1, a locus of Arabidopsis thaliana that is required for disease resistance to both a bacterial and a fungal pathogen. Proc Natl Acad Sci USA 92:6597-6601

Clough SJ, Bent AF (1998) Floral dip:a simplified method for Agrobacterium-mediated transformation of Arabidopsis thaliana. Plant J 16:735-743

Frye CA, Tang D, Innes RW (2001) Negative regulation of defense responses in plants by a conserved MAPKK kinase. Proc Natl Acad Sci USA 98:373-378

Gepstein S (2004) Leaf senescence-not just a 'wear and tear' phenomenom. Genome Biol 5:212

Glazebrook J (2005) Contrasting mechanisms of defense against biotrophic and necrotrophic pathogens. Annu Rev Phytopathol 43:205-227

Govrin EM, Levine A (2000) The hypersensitive response facilitates plant infection by the necrotrophic pathogen Botrytis cinerea. Curr Biol 10:751-757

van der Graaff E, Schwacke R, Schneider A, Desimone M, Flugge UI, Kunze R (2006) Transcription analysis of Arabidopsis membrane transporters and hormone pathways during developmental and induced leaf senescence. Plant Physiol 141:776-792

Grbic V, Bleecker AB (1995) Ethylene regulates the timing of leaf senescence in Arabidopsis. Plant J 8:595-602

Guo Y, Gan S (2005) Leaf senescence: signals, execution, and regulation. Curr Top Dev Biol 71:83-112

Guo Y, Gan S (2006) AtNAP, a NAC family transcription factor, has an important role in leaf senescence. Plant $\mathrm{J}$ 46:601-612

Guo Y, Cai Z, Gan S (2004) Transcriptome of Arabidopsis leaf senescence. Plant Cell Environ 27:521-549
Hanfrey C, Fife M, Buchanan-Wollaston V (1996) Leaf senescence in Brassica napus: expression of genes encoding pathogenesis-related proteins. Plant Mol Biol 30:597-609

He Y, Fukushiga H, Hildebrand DF, Gan S (2002) Evidence supporting a role of jasmonic acid in Arabidopsis leaf senescence. Plant Physiol 128:876-884

Jefferson RA, Kavanagh TA, Bevan MW (1987) GUS fusions: $\beta$-glucuronidase a sensitive and versatile gene fusion marker in higher plants. EMBO J 6:3901-3907

John CF, Morris K, Jordan BR, Thomas B, A-H-Mackerness S (2001) Ultraviolet-B exposure leads to up-regulation of senescence-associated genes in Arabidopsis thaliana. J Exp Bot 52:1367-1373

Kieber JJ, Rothenberg M, Roman G, Feldmann KA, Ecker JR (1993) CTR1, a negative regulator of the ethylene response pathway in Arabidopsis, encodes a Raf family of protein kinases. Cell 72:427-441

Koncz C, Schell J (1986) The promotor of TL-DNA gene 5 controls the tissue-specific expression of chimaeric genes carried by a novel type of Agrobacterium binary vector. Mol Gen Genet 204:383-396

Kus JV, Zaton K, Sarkar R, Cameron RK (2002) Age-related resistance in Arabidopsis is a developmentally regulated defense response to Pseudomonas syringae. Plant Cell 14:479_ 490

La Camera S, Geoffroy P, Samaha H, Ndiaye A, Rahim G, Legrand M, Heitz T (2005) A pathogen-inducible patatinlike lipid acyl hydrolase facilitates fungal and bacterial host colonization in Arabidopsis. Plant J 44:810-825

Li J, Brader G, Palva ET (2004) The WRKY70 transcription factor: a node of convergence for jasmonate-mediated and salicylate-mediated signals in plant defense. Plant Cell 16:319-331

Li J, Brader G, Kariola T, Tapio Palva E (2006) WRKY70 modulates the selection of signaling pathways in plant defense. Plant J 46:477-491

Lin J-F, Wu S-H (2004) Molecular events in senescing Arabidopsis leaves. Plant J 39:612-628

Maleck K, Levine A, Eulgem T, Morgen A, Schmid J, Lawton K, Dangl JL, Dietrich RA (2000) The transcriptome of Arabidopsis thaliana during systemic acquired resistance. Nat Genet 26:403-410

Miao Y, Laun T, Zimmermann P, Zentgraf U (2004) Targets of the WRKY53 transcription factor and its role during leaf senescence in Arabidopsis. Plant Mol Biol 55:853-867

Morris K, A-H-Mackerness S, Page T, John CF, Murphy AM, Carr JP, Buchanan-Wollaston V (2000) Salicylic acid has a role in regulating gene expression during leaf senescence. Plant J 23:677-685

Oh SA, Lee SY, Chung IK, Lee C-H, Nam HG (1996) A senescence-associated gene of Arabidopsis thaliana is distinctively regulated during natural and artificially induced leaf senescence. Plant Mol Biol 30:739-754

Oh SA, Park JH, Lee GI, Paek KH, Park SK, Nam HG (1997) Identification of three genetic loci controlling leaf senescence in Arabidopsis thaliana. Plant J 12:527-535

Olsen AN, Ernst HA, Leggio LL, Skriver K (2005) NAC transcription factors: structurally distinct, functionally diverse. Trends Plant Sci 10:79-87

Park J-H, Halitschke R, Kim HB, Baldwin IT, Feldmann K, Feyereisen R (2002) A knock-out mutation in allene oxide synthase results in male sterility and defective wound signal transduction in Arabidopsis due to a block in jasmonic acid biosynthesis. Plant J 31:1-12

Quirino BF, Noh Y-S, Himelblau E, Amasino RM (2000) Molecular aspects of leaf senescence. Trends Plant Sci 5:278-282 
Robatzek S, Somssich IE (2001) A new member of the Arabidopsis WRKY transcription factor family, AtWRKY6, is associated with both senescence- and defense-related processes. Plant J 28:123-133

Rosso MG, Li Y, Strizhov N, Reiss B, Dekker K, Weisshaar B (2003) An Arabidopsis thaliana T-DNA mutagenized population (GABI-Kat) for flanking sequence tag-based reverse genetics. Plant Mol Biol 53:247-259

Schenk PM, Kazan K, Rusu AG, Manners JM, Maclean DJ (2005) The SEN1 gene of Arabidopsis is regulated by signals that link plant defence responses and senescence. Plant Physiol Biochem 43:997-1005

Schmid M, Davison TS, Henz SR, Pape UJ, Demar M, Vingron M, Scholkopf B, Weigel D, Lohmann JU (2005) A gene expression map of Arabidopsis thaliana development. Nat Genet 37:501

Swidzinski JA, Sweetlove LJ, Leaver CJ (2002) A custom microarray analysis of gene expression during programmed cell death in Arabidopsis thaliana. Plant J 30:431-446

Tang D, Innes RW (2002) Overexpression of a kinase-deficient form of the EDR1 genes enhances powdery mildew resistance and ethylene-induced senescence in Arabidopsis. Plant J 32:975-983

Ward ER, Uknes SJ, Williams SC, Dincher SS, Wiederhold DL, Alexander DC, Ahl-Goy P, Métraux J-P, Ryals JA (1991) Coordinate gene activity in response to agents that induce systemic acquired resistance. Plant Cell 3:10851094
Weaver LM, Amasino RM (2001) Senescence is induced in individually darkened Arabidopsis leaves, but inhibited in whole darkened plants. Plant Physiol 127:876-886

Wyatt S, Pan S, Kuc J (1991) Beta-1,3-Glucanase, chitinase and peroxidase activities in tobacco tissues resistant and susceptible to blue mould as related to flowering, age and sucker development. Physiol Mol Plant Pathol 39:433-440

Yoshida S, Ito M, Nishida I, Watanabe A (2001) Isolation and RNA gel blot analysis of genes that could serve as potential molecular markers for leaf senescence in Arabidopsis thaliana. Plant Cell Physiol 42:170-178

Yoshida S, Ito M, Callis J, Nishida I, Watanabe A (2002a) A delayed leaf senescence mutant is defective in arginyltRNA:protein arginyl transferase, a component of the N-end rule pathway in Arabidopsis. Plant J 32:129-137

Yoshida S, Ito M, Nishida I, Watanabe A (2002b) Identification of a novel gene $H Y S 1 / C P R 5$ that has a repressive role in the induction of leaf senescence and pathogen-defense responses in Arabidopsis thaliana. Plant J 29:427-437

Zimmermann P, Hirsch-Hoffmann M, Hennig L, Gruissem W (2004) GENEVESTIGATOR. Arabidopsis microarray database and analysis toolbox. Plant Physiol 136:2621-2632

Zimmermann P, Hennig L, Gruissem W (2005) Gene-expression analysis and network discovery using Genevestigator. Trends Plant Sci 10:407-409

Ülker B, Somssich IE (2004) WRKY transcription factors: from DNA binding towards biological function. Curr Opin Plant Biol 7:491-498 